\title{
Utilizing Three-dimensional Printing in treating Challenged Dental Implant Cases
}

\author{
Abdullah Alodadi
}

\section{ABSTRACT}

Background: Congenital missing laterals are considered a quite common dental abnormality. The limited arch length makes implant procedure sometimes very challenging to be considered as a restorative option.

Aim: This case report aimed to demonstrate the benefit of utilizing three-dimensional (3D) printed models in managing challenging implant cases in limited arch length for congenitally missing maxillary lateral incisors.

Case report: A 39-year-old female Afro-American patient presented at the Ashman Department of Periodontology and Implant Dentistry, New York University College of Dentistry, to replace her congenitally missing maxillary laterals. Adequate mesiodistal arch length was present for edentulous areas of \#12 and 22 clinically. Cone beam computed tomography (CBCT) showed adequate buccolingual bone width. Three-dimensional printed models were used to evaluate the labial angulation of the alveolar ridge, facial concavities and to demonstrate the surgical implant placement prior to the day of surgery. Bonelevel tapered implant with $3.3 \mathrm{~mm}$ diameter and $12 \mathrm{~mm}$ length (StraumannRoxolid ${ }^{\circledR}$ SLActive ${ }^{\circledR}$ implant) was placed in palatal position. Immediate cement retained nonfunctional provisional crown, fabricated, cemented on a prefabricated abutment to provide soft tissue contouring and immediate esthetic restoration. One week later, the same surgical protocol was followed in the area of missing \#12.

Conclusion: Placement of dental implant in limited mesiodistal edentulous space in an esthetic zone is a challenging procedure that requires thorough assessment and adequate surgical and restorative expertise to achieve optimum outcome. The 3D printing has proved its valuable benefit in treatment planning and executing difficult implant treatment by visualizing the supporting tissues in spatial dimensions and providing accurate measurements and in demonstrating hands on before the surgery time.

Clinical significance: The 3D visualization (using CBCT) allows for rapid site analysis and predictable treatment planning in various difficult cases of implant placement, especially in cases of congenitally missing maxillary lateral incisors with less arch length.

Keywords: Esthetics, Implants, Provisionalization.

Department of Prosthodontics and Implant Dentistry, Dental Center, Armed Forces Hospitals Southern Region, Khamis Mushait, Kingdom of Saudi Arabia

Corresponding Author: Abdullah Alodadi, Department of Prosthodontics and Implant Dentistry, Dental Center, Armed Forces Hospitals Southern Region, Khamis Mushait, Kingdom of Saudi Arabia, e-mail: abdullahalodadi@gmail.com
How to cite this article: Alodadi A. Utilizing Three-dimensional Printing in treating Challenged Dental Implant Cases. World J Dent 2018;9(3):235-241.

Source of support: Nil

Conflict of interest: None

\section{BACKGROUND}

Congenitally missing lateral incisors are a quite common dental abnormality. It has been reported with $22 \%$ incidence, which is slightly more in females. ${ }^{1}$ The normal mesiodistal dimension of maxillary laterals is $5.5 \mathrm{~mm}$ with an average of 4.5 to $6.7 \mathrm{~mm}$. This limited arch length makes implant procedure sometimes very challenging to be considered as a restorative option, considering that the minimal tooth to implant distance should be $1.5 \mathrm{~mm}$ to maintain interproximal bone and soft tissue. ${ }^{2}$

Implant placement in the esthetic region is a complex clinical procedure and requires a restorative-driven approach. Proper selection of patients and implant system together with thorough assessment of the risk of esthetic complications are of utmost importance. Correct 3D implant positioning and sufficient bone volume should provide long-term esthetic and functional benefits. ${ }^{3,4}$ Esthetic zone is the area where the clinician should anticipate the risk of complications that may arise due to the presence of thin scalloped gingival biotype or inadequate bone width or height. Implant placement in esthetic zone is particularly challenging. Recently, computer software allows the user to utilize the CBCT DICOM data during radiographic interpretation that permits rendering of the bone volumes before surgical procedures. Moreover, it allows for more precise implantsite measurement. ${ }^{5,6}$

Positioning of single implant within the dental arch is challenging, considering the proximity to adjacent tooth roots, vital structures, occlusal plane, and relative position within the arch. When positioning an implant, digital software will allow the user to place a virtual analog of the proposed implant and measure the optimum distance between the previously mentioned structures (Fig. 1). However, to achieve successful esthetic outcome for a patient with high esthetic expectations, immediate implant provisionalization protocol is preferred. Replacing single tooth with dental implant in an immediate provisionalization protocol is considered as a viable and 


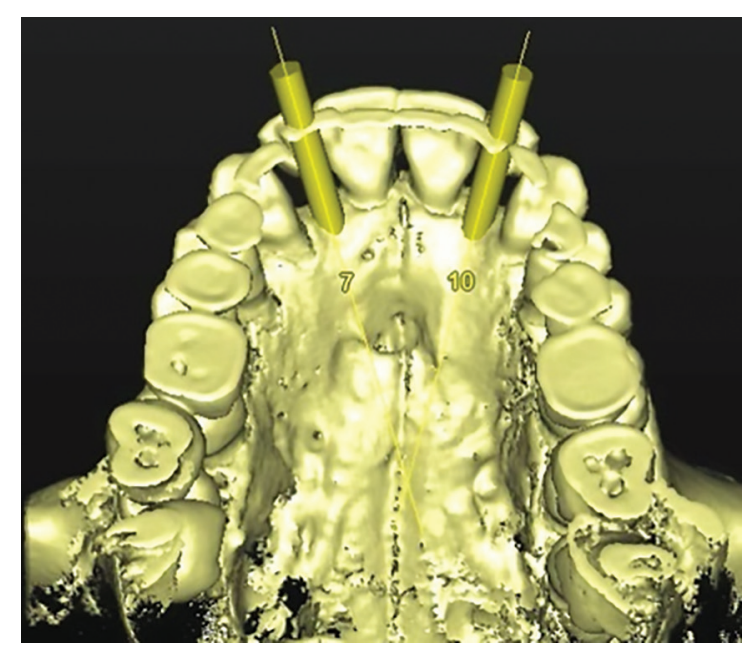

Fig 1: Simulated implant position utilizing CBCT

safe treatment option for both maxilla and mandible. ${ }^{7}$ Chrcanovic et $\mathrm{al}^{8}$ reported in a systematic review and meta-analysis that the differences in occlusal loading between immediate nonfunctional loading and immediate functional loading might not affect the survival of dental implants and that there is no apparent significant effect on the marginal bone loss.

\section{CASE REPORT}

A 39-year-old female Afro-American patient presented at the Ashman Department of Periodontology and Implant
Dentistry, New York University College of Dentistry, to replace her congenitally missing maxillary laterals. Patient had undergone orthodontic treatment recently to correct her malaligned teeth for a period of 3 years. Patient was in a good medical health with no absolute/ relative contraindication for dental implant surgery (Fig. 2A).

Adequate mesiodistal arch length was present for edentulous areas of \#12 and 22 clinically (Fig. 2B). Thick and flat gingival biotype and fair oral hygiene was maintained (Fig. 2C). Cone beam CT shows adequate buccolingual bone width (Fig. 3). Nevertheless, root proximity was noticed between laterals and canines. Narrow-diameter implants could be considered as one of the restorative options for limited interdental space. ${ }^{9}$ The use of transitional implants for immediate fixed temporary prostheses has demonstrated successful outcome documented in the literature. ${ }^{10}$ According to Buser et al, ${ }^{3}$ implant shoulder should be at least $1.5 \mathrm{~mm}$ away from the root of the adjacent tooth in the mesiodistal dimension in order to prevent resorption of the interproximal alveolar crest. Implant shoulder should be positioned about $1 \mathrm{~mm}$ apically to the cementoenamel junction of the adjacent teeth. In the orofacial dimension, implant shoulder should be positioned about 1.5 to $2.0 \mathrm{~mm}$ palatally from the imaginary line connecting the point of emergence of adjacent teeth. Positioning dental implant midway
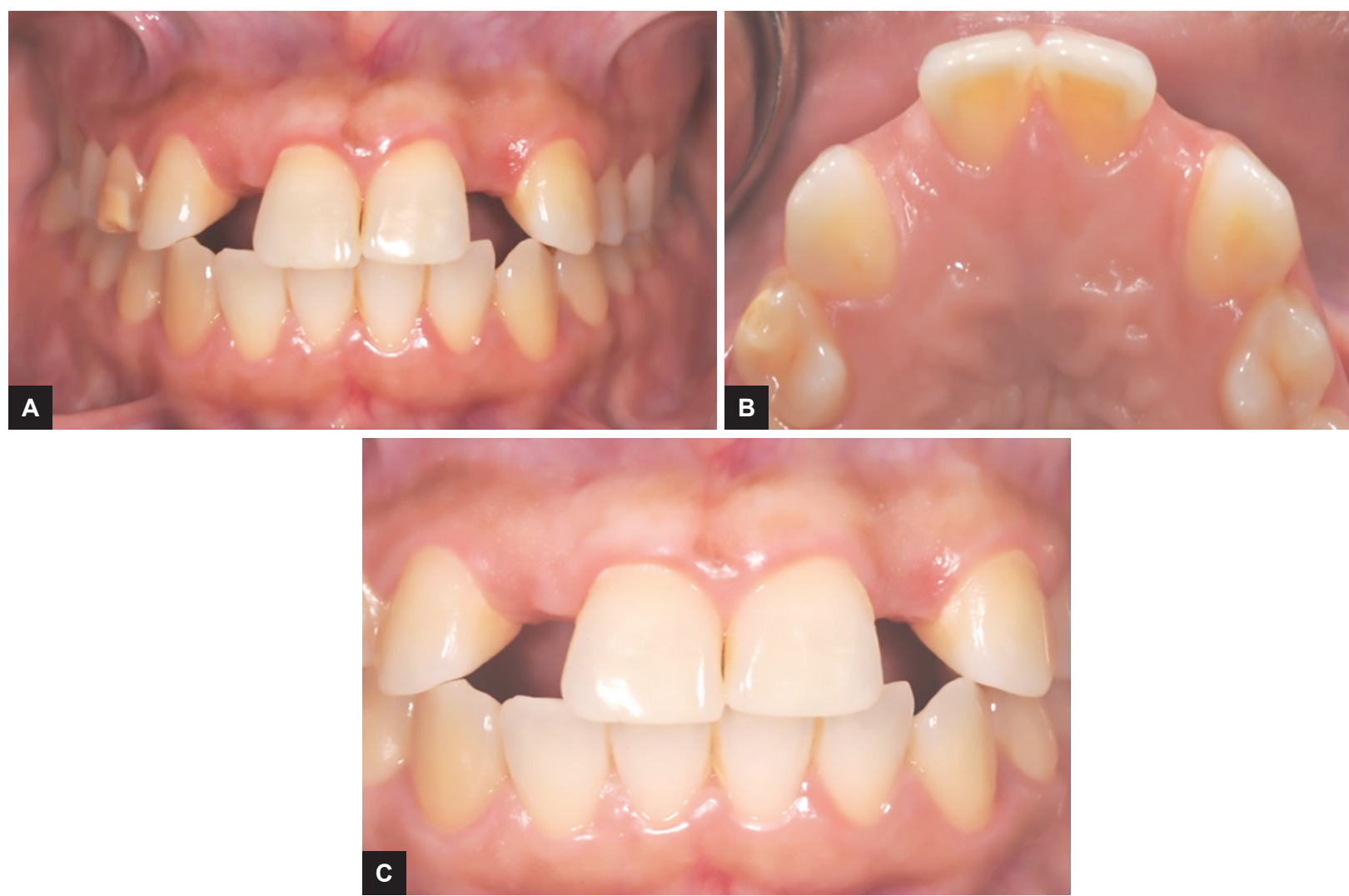

Figs $2 A$ to C: (A) Preoperative clinical retracted frontal view; (B) clinical occlusal view shows enough clinical mesiodistal length; and (C) a close-up frontal view 


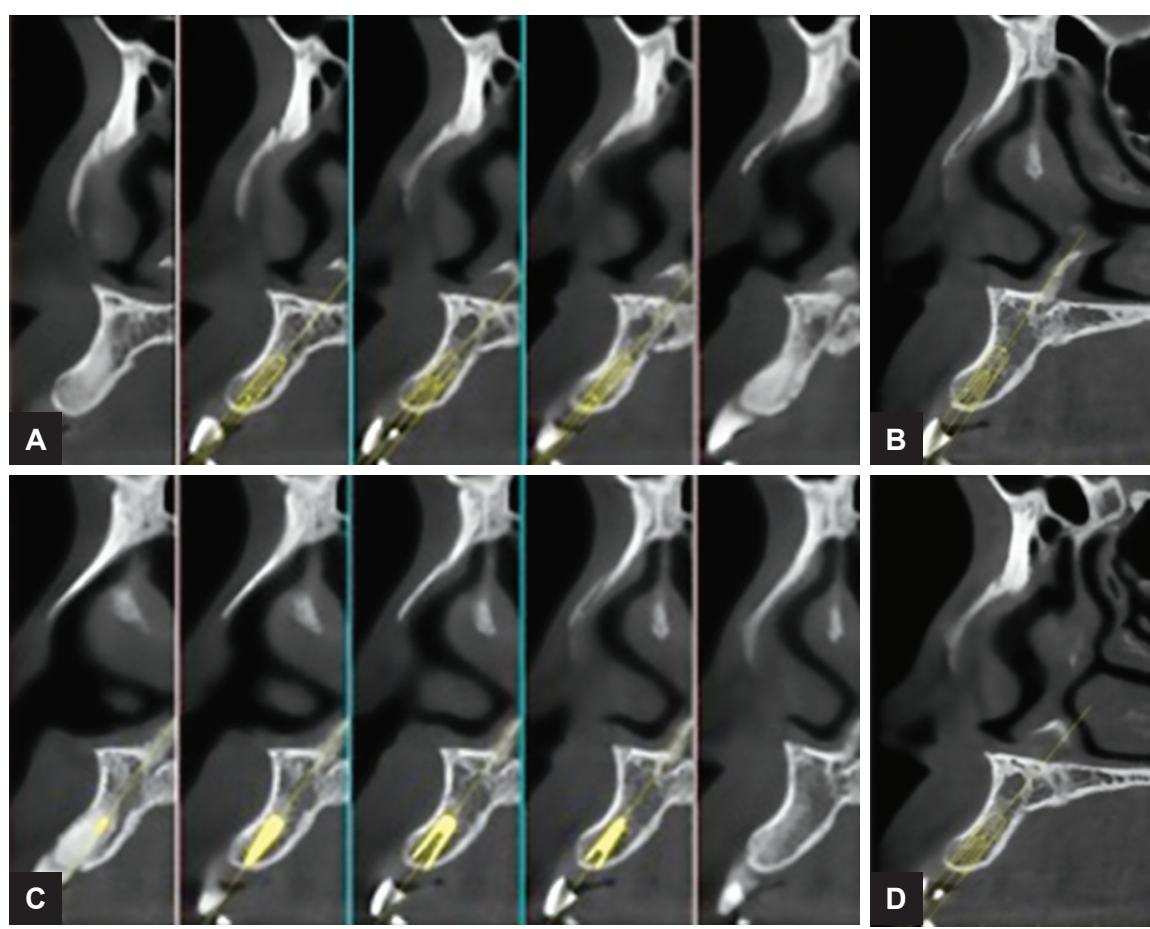

Figs 3A to D: Radiographic sagittal cross-section view for both sites of \#12 and 22; notice the adequate buccolingual ridge width

between the buccal and lingual cortical plate in our case will result in damaging the roots of the neighboring teeth (Fig. 4A). However, patient denied undergoing another orthodontic treatment to align and achieve roots parallelism. Therefore, 3D printed model was used to evaluate the labial angulation of the alveolar ridge, facial concavities and to demonstrate the surgical implant placement before the actual day of surgery (Fig. 4B).

Based on all the available diagnostic tools, treatment plan was formulated and consisted of cement retained all-ceramic crowns retained with narrow diameter dental implants. Implant labiolingual position was critical; it was decided to place the implant in more palatal position along with the angulation of the alveolar ridge (Figs 4C and D).

\section{Surgical and Prosthetic Phase}

Pre- and postsurgical medication regimen was received by the patient as follows: antibiotics presurgery and 5 days postsurgery (amoxicillin $500 \mathrm{mg}$ ); cortisone medication (prednisone $5 \mathrm{mg}$ ), given daily in a regression mode (25-10 mg) from the day of surgery until 4 days postoperatively; anti-inflammatory medication (ibuprofen $600 \mathrm{mg}$ ), administered for 3 days postoperatively. The surgery was performed under local anesthesia (lidocaine hydrochloride $2 \%$ with epinephrine 1:100,000).

Midcrestal incision and sulcular incision was performed in edentulous area of \#22 and full-thickness flap reflected (Fig. 5A). Bone-level tapered implant with $3.3 \mathrm{~mm}$ diameter and $12 \mathrm{~mm}$ length (StraumannRoxolid ${ }^{\circledR}$ SLActive ${ }^{\circledR}$ implant) was placed in palatal position (Fig. 5B). Implant position was evaluated using periapical radiographs to ensure maintaining space between dental implant and neighboring teeth (Fig. 5C). Immediate cement-retained nonfunctional provisional crown, fabricated chair side, was cemented on a prefabricated abutment to provide soft tissue contouring and immediate esthetic restoration (Fig. 6A). One week later, the same surgical protocol was followed in the area of missing \#12. Midcrestal incision was located more toward the palatal position to manipulate the keratinized tissue in the facial aspect, full-thickness flap reflected, and bone-level tapered implant with $3.3 \mathrm{~mm}$ diameter and $12 \mathrm{~mm}$ length (StraumannRoxolid ${ }^{\circledR}$ SLActive ${ }^{\circledR}$ implant) was placed in palatal position (Figs 6B, C and 7A). Then chair-side fabricated provisional crown was cemented on a prefabricated abutment (Fig. 7B). One week follow-up for implant in position of \# 22 showed excellent soft tissue healing (Fig. 7C).

After 2 months of a stable healing period, provisional crowns were detached and implant level impression made. Computer-aided design/computed-aided manufacturing-fabricated zirconium abutments were fabricated to provide the maximum esthetics and strength (Fig. 8). After removal of the provisional restoration and temporary abutment, the final abutment was secured. Once the fit was assessed radiographically, the abutment screw was torqued to $35 \mathrm{~N} \mathrm{~cm}$. The screw access holes were obturated with a light-cured temporary restorative 

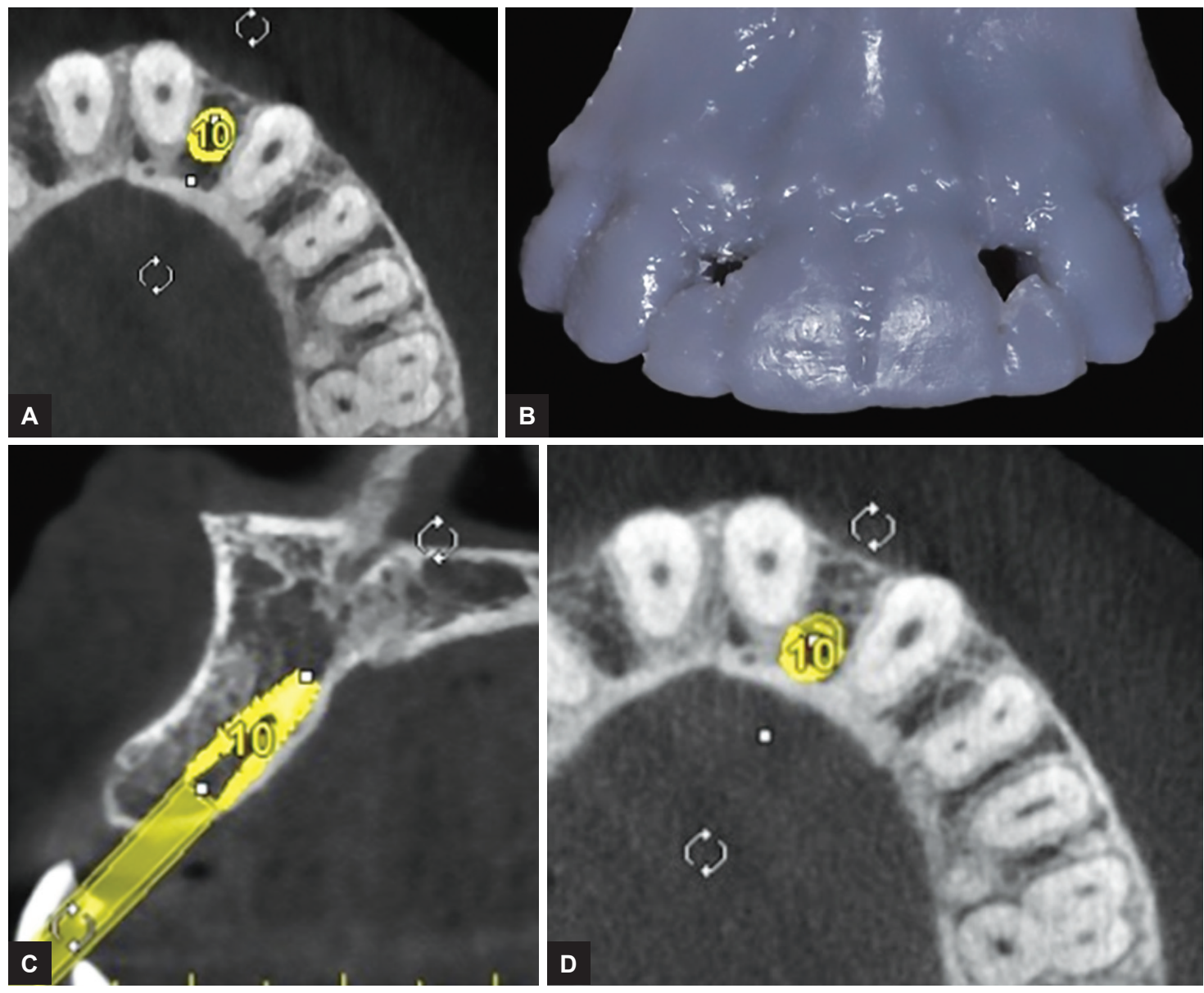

Figs 4A to D: (A) Radiographical coronal section shows how close the implant will be if placed in the middle of the buccolingual dimension of the ridge; (B) a printed 3D model; (C) implant instead will be placed in palatal position; and $(D)$ this new position will allow to avoid injuring the roots of neighboring teeth
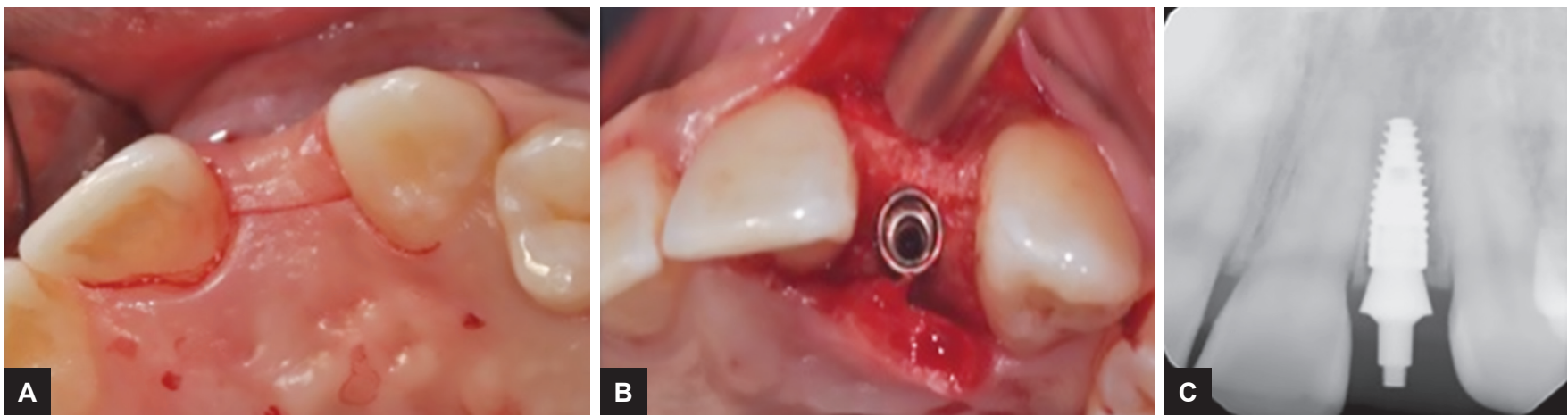

Figs 5A to C: (A) Midcrestal incision \#22; (B) implant placement \#22; and (C) periapical radiograph shows the intermediate position of the implant between the roots
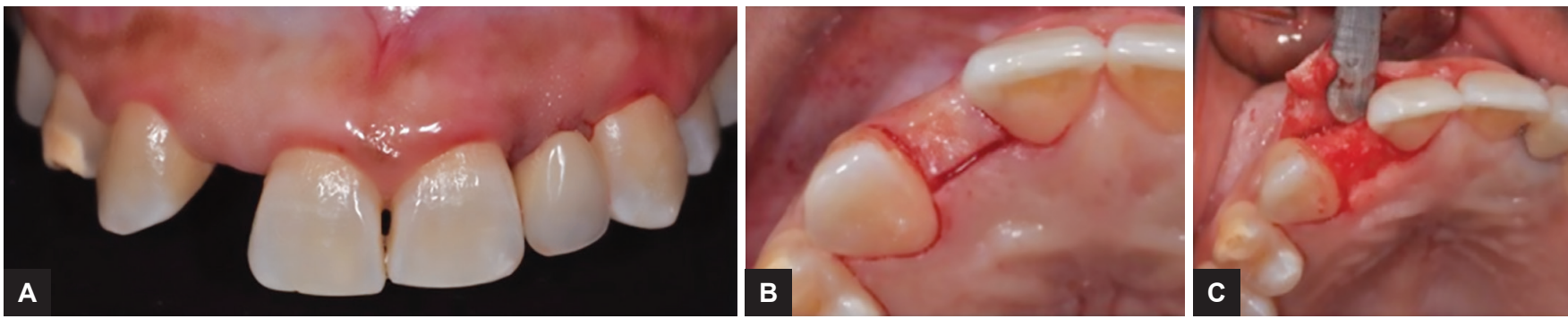

Figs 6 A to C: (A) Frontal retracted view for the immediate nonfunctional provisional on \#22; (B) midcrestal incision \#12; and $(C)$ full-thickness flap reflection \#12 

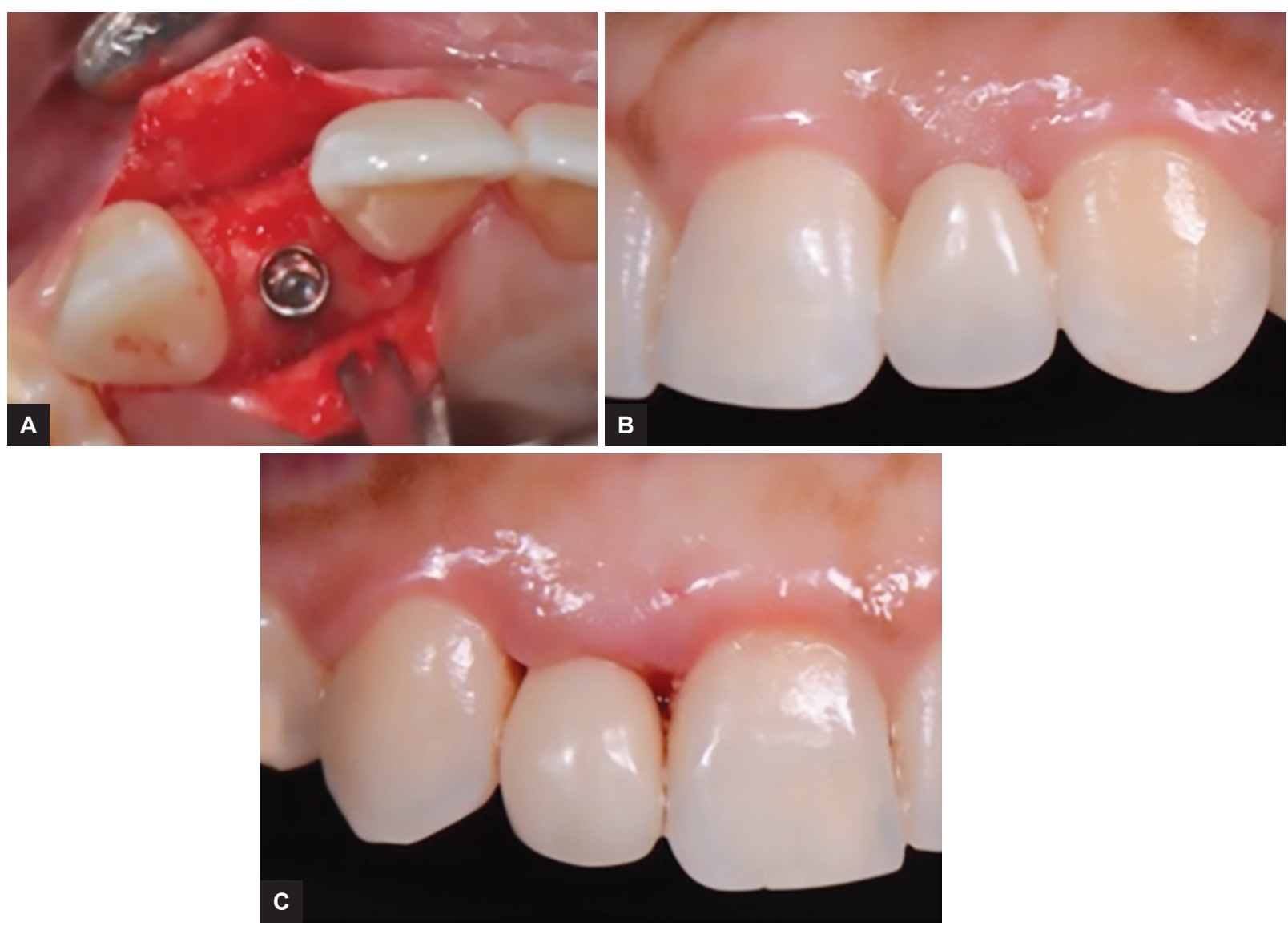

Figs 7A to C: (A) Implant placement \#12; (B) immediate implant provisionalization \#12; and (C) 1 week follow-up of \#22
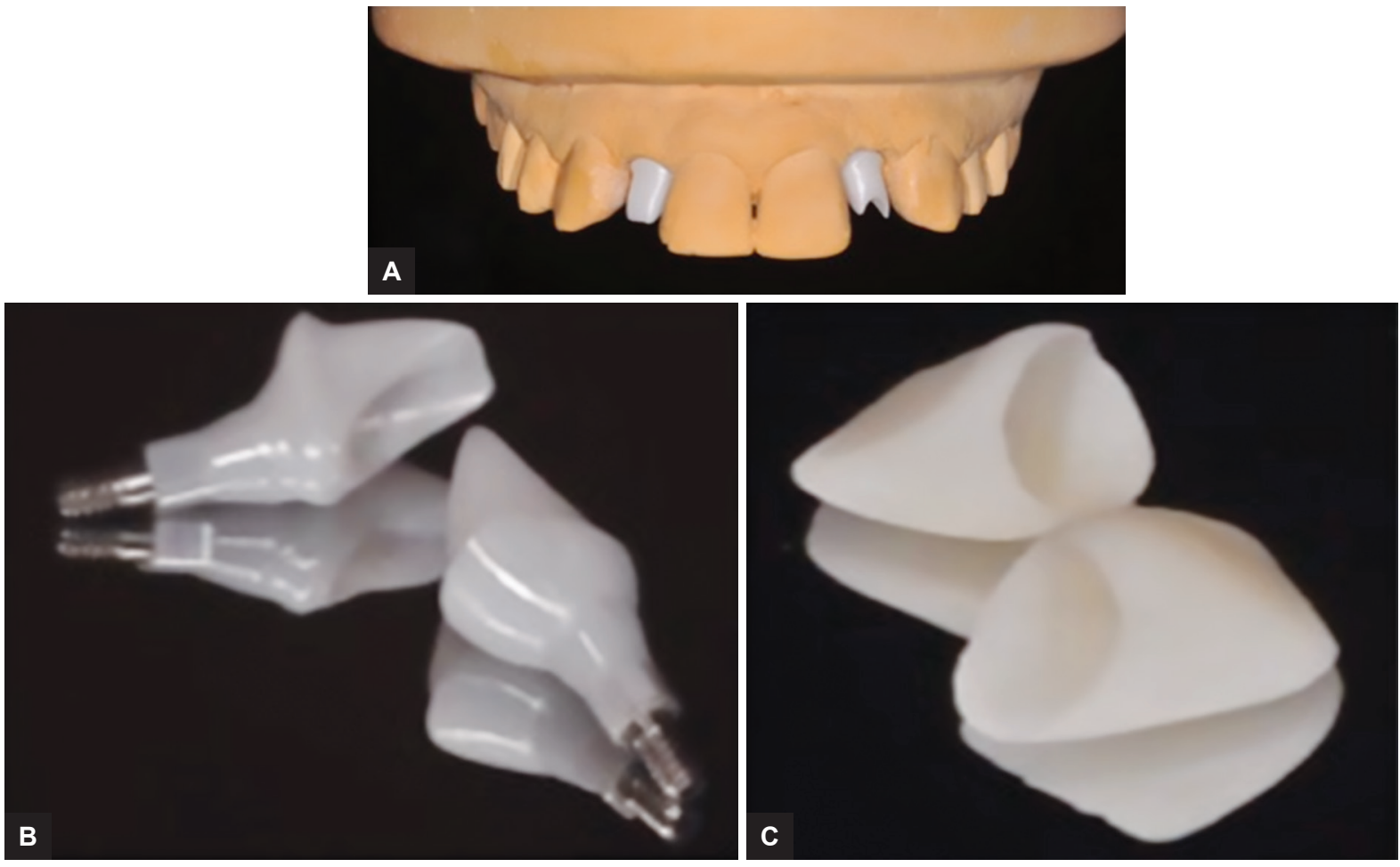

Figs 8A to C: Fabricated zirconium abutments; zirconium single crowns 

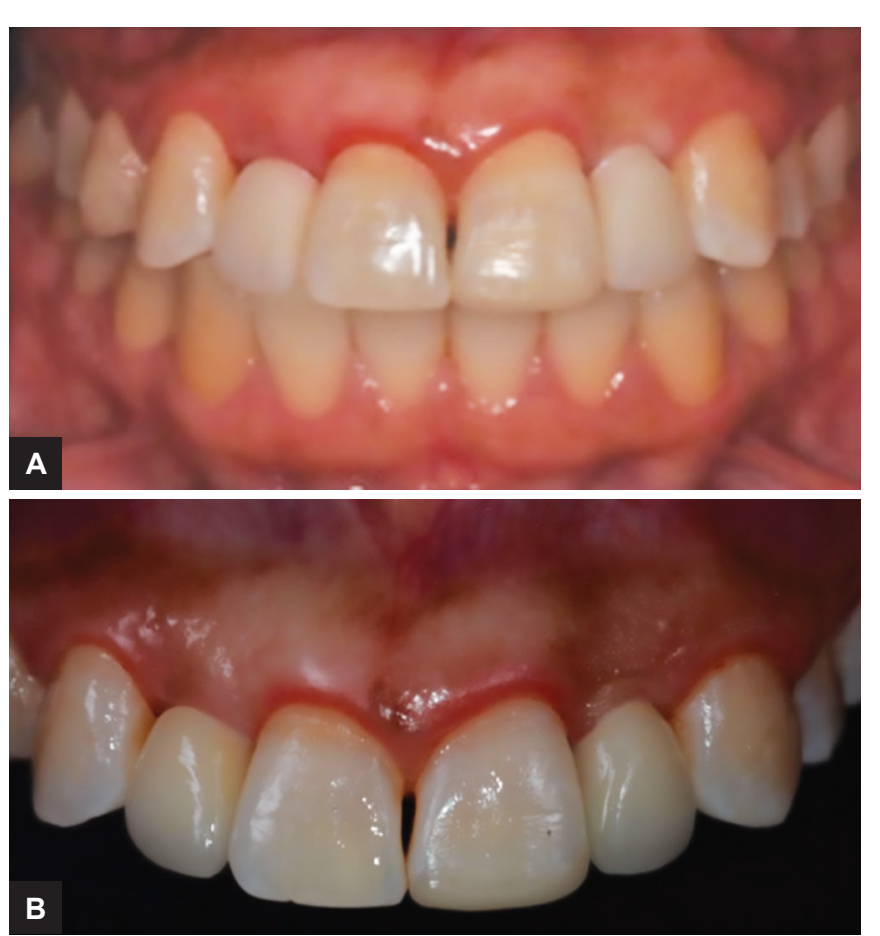

Figs 9A and B: (A) Bisque try-in; and (B) final zirconium crowns after final cementation

material. Then zirconium crown was tried and adjusted with abrasives to achieve adequate crown contour and natural looking anatomy (Fig. 9A). After having the crowns glazed, the crowns were then cemented using resin-modified glass ionomer cement (Fig. 9B).

\section{DISCUSSION}

Maxillary lateral incisors are the most common congenitally missing teeth after upper and lower second premolars. ${ }^{11}$ Treatment options include orthodontic movement of cuspids into lateral incisor sites, prosthodontic restorations including fixed and removable prostheses and resinbonded retainers, and single tooth implants. ${ }^{12}$ In various cases, the patients do not wish to undergo orthodontic treatment as in the present case.

In this case, 3D model was printed to evaluate the labial angulation of the alveolar ridge, facial concavities and demonstrate the surgical implant placement before the actual surgery took place.

Such a 3D visualization allows for rapid site analysis and predictable treatment planning, whereby the surgeon can order specific implant diameters and sizes, healing abutments, and provisional crowns. With the advancement of technology, 3D stereolithographics will be of a great help in visualizing the anatomy of the site of operation, demonstrating the surgical procedure beforehand and in aiding in fabrication of an accurate surgical guide. Correct 3D implant positioning and sufficient bone volume provide long-term benefits in terms of esthetics and function. ${ }^{3}$
It has been mentioned in previous studies that $\mathrm{CBCT}$ provides much precise and accurate dimensions, which was not so in two-dimensional imaging methods. It makes it possible for the clinicians to combine the ideas from multidisciplinary team so as to make a single perfect treatment plan to get the best treatment outcome. Moreover, CBCT has been quite beneficial for optimal treatment planning by reducing the associated risks to a great extent. ${ }^{13}$

\section{CONCLUSION}

Placement of dental implant in limited mesiodistal edentulous space in an esthetic zone is a challenging procedure that requires thorough assessment and adequate surgical and restorative expertise to achieve optimum outcome. The 3D printing has proved its valuable benefit in treatment planning and executing difficult implant treatment by helping in visualizing the supporting tissues in spatial dimensions and providing accurate measurements and in demonstrating it hands-on before the surgery.

\section{CLINICAL SIGNIFICANCE}

The 3D visualization (using $\mathrm{CBCT}$ ) allows for rapid site analysis and predictable treatment planning in various difficult cases of implant placement, especially in cases of congenitally missing maxillary lateral incisors with less arch length.

\section{REFERENCES}

1. Polder BJ, Van't Hof MA, Van der Linden FP, KuijpersJagtman AM. A meta-analysis of the prevalence of dental agenesis of permanent teeth. Community Dent Oral Epidemiol 2004 Jun;32(3):217-226.

2. Tarnow D, Elian N, Fletcher P, Froum S, Magner A, Cho SC, Salama M, Salama H, Garber DA. Vertical distance from the crest of bone to the height of the interproximal papilla between adjacent implants. J Periodont 2003 Dec;74(12): 1785-1788.

3. Buser, D.; Belser, U.; Wismcijer, D. Implant therapy in the esthetic zone-single tooth replacements. Berlin: Quintessence Publishing; 2007.

4. Chen, S.; Buser, D. Implant placement in post-extraction sitestreatment options. Berlin: Quintessence Publishing; 2008.

5. Scherer MD. Presurgical implant-site assessment and restoratively driven digital planning. Dent Clin North Am 2014 Jul;58(3):561-595.

6. Mora MA, Chenin DL, Arce RM. Software tools and surgical guides in dental-implant-guided surgery. Dent Clin North Am 2014 Jul;58(3):597-626.

7. Malo P, de Araujo Nobre M, Lopes A, Ferro A, Gravito I. Single-tooth rehabilitations supported by dental implants used in an immediate- provisionalization protocol: report on long-term outcome with retrospective follow-up. Clin Implant Dent Relat Res 2015 Oct;17(Suppl 2):e511-e519. 
8. Chrcanovic BR, Albreksson T, Wenneberg A. Immediate nonfunctional versus immediate functional loading and dental implant failure rate: a systematic review and meta-analysis. J Dent 2014 Sep;42(9):1052-1059.

9. Froum S, Emtiaz S, Bloom MJ, Scolnick J, Tarnow DP. The use of transitional implants for immediate fixed temporary prostheses in cases of implant restorations. Pract Periodontics Aesthet Dent 1998 Aug;10(6):737-746.

10. Froum SJ, ChoSC, Cho YS, Elian N, Tarnow D. Narrow-diameter implants: a restorative option for limited interdental space. Int J Periodontics Restorative Dent 2007 Oct;27(5):449-455.
11. Abu-Hussein M, Watted N, Watted A, Abu-Hussein Y, Yehia M. Awadi O, Azzaldeen A. Prevalence of tooth agenesis in orthodontic patients at Arab Population in Israel. Int J Public Health Res 2015 Apr;3(3):77-82.

12. Muhamad AH, Azzaldeen A, Nezar W, Mohammed Z. Esthetic evaluation of implants placed after orthodontic treatment in patients with congenitally missing lateral incisors. J Adv Med Dent Sci Res 2015 Jul-Sep;3(3):110-118.

13. Worthington P, Rubenstein J, Hatcher DC. The role of conebeam computed tomography in the planning and placement of implants. J Am Dent Assoc 2010 Oct;141(Suppl 3):19S-24S. 